\title{
Large-amplitude oscillations and chaos in a Hamiltonian plasma system with many degrees of freedom
}

\author{
Daniela Farina ${ }^{1}$ and Roberto Pozzoli ${ }^{2}$ \\ ${ }^{1}$ Istituto di Fisica del Plasma, Consiglio Nazionale delle Ricerche, EURATOM-ENEA-CNR Association, via Cozzi 53, \\ 20125 Milano, Italy \\ ${ }^{2}$ Istituto Nazionale di Fisica Nucleare and Istituto Nazionale di Fisica della Materia, Università degli Studi di Milano, \\ Dipartimento di Fisica, via Celoria 16, 20133 Milano, Italy
}

(Received 6 December 2003; published 27 September 2004)

\begin{abstract}
Taking the beam-plasma system as a reference Hamiltonian system with many degrees of freedom, the connection between the development of large amplitude oscillations and stochasticity of the system (measured through the time-dependent maximum Lyapunov exponent) is investigated. It is found that the development of self-consistent large amplitude oscillations occurs in correspondence with the onset of chaos, and is related to a well defined change of topology of the phase space of the system. It is also shown that in a (Hamiltonian) linearly stable regime the development of large amplitude oscillations can occur when weakly dissipative processes are introduced.
\end{abstract}

DOI: 10.1103/PhysRevE.70.036407

PACS number(s): 52.35.- $\mathrm{g}, 05.45 .-\mathrm{a}, 52.25 . \mathrm{Gj}$

\section{INTRODUCTION}

The nonlinear development of a perturbation in a collisionless plasma is a topic thoroughly investigated in the literature, both analytically and numerically [1-3]. Even in its simplest, one-dimensional, formulation, it can reveal new features, which happen to be of interest for a variety of physical systems, whenever these features derive from the quite generic Hamiltonian structure of the system, as in the present case. Here, we refer explicitly to a beam-plasma system, which can be considered a paradigm for the study of plasma-wave interaction and vortex formation in phase space, and perform a Hamiltonian analysis, which can therefore be directly extended to other systems, e.g., Compton FEL [4], self-interacting stars [5], interacting vortices in 2D fluids [6], the common link among them being the structure of the $N$ - particle Hamiltonian.

The system is initially very close to an equilibrium condition where the field is almost zero, and energy and momentum mostly reside in the particles. It is then followed during its whole evolution, looking for conditions where selfsustained large oscillations of the field are produced. This process is studied in the literature using either the full Vlasov-Poisson system, or the corresponding Hamiltonian model, in which the beam is represented by an ensemble of $N$ particles. It is found that a single parameter $\Delta$ characterizes the system evolution: when its value is larger than a well defined critical value $\Delta_{c}$ (linear or stable regime) the field amplitude exhibits small periodic deviations from its initial value during the whole evolution; when $\Delta<\Delta_{c}$ (nonlinear or "unstable" regime) large amplitude oscillations of the field occur on the long time scale. Note that, as far as the linear analysis is valid, the motion of the $\mathrm{N}$-particle system is fully integrable, and stochasticity is absent: this applies both to the stable regime and to the initial stage of the unstable regime. Therefore, stochasticity, if arises, can occur only in the fully nonlinear regime. Here, stochasticity refers to the $N$ particle system representing the beam, i.e., to the motion of the rep- resentative point in the $2 \mathrm{~N}$-dimensional phase space, the degree of stochasticity of the system being measured by the value of the largest Lyapunov exponent. The aim of the present paper is to find the relation between the onset of stochasticity in the dynamics of the system and the transition to a nonlinear stage characterized by large field amplitude oscillations.

The main result is the following: the development of self consistent large oscillations entails the occurrence of stochasticity, i.e., starting from a condition with vanishingly small field, large oscillations can be excited in the Hamiltonian system only when stochasticity arises. This process is connected to a topological transition in the $2 \mathrm{~N}$-dimensional phase space of the beam. Such a transition can occur even when the system is initially linearly stable, if a dissipative mechanism is taken into account, which relaxes the Hamiltonian constraints. We show that this occurs in the system under consideration, when radiation damping or frictional effects are introduced.

The phase space analysis of the dynamics of the system is presented in Sec. II. The analysis of stochasticity and the role of weakly dissipative effects are presented in Sec. III. The last section is devoted to short concluding remarks.

\section{PHASE SPACE ANALYSIS}

The system is made up of a monoenergetic beam, of density $n_{b}$ and velocity $V_{b}$, represented by $N$ particles; a Maxwellian electron bulk, of density $n_{0}$, which responds linearly to the field, through the dielectric permittivity $\epsilon(k, \omega)$, and a cold, uniform ion background, providing charge neutrality. The electric field is determined self-consistently by the Poisson equation, and is initially present as a seed, i.e., as a plasma wave with very small amplitude. Phase velocities much larger than the electron thermal speed are assumed [3]. The beam distribution function can be written as $f_{b}$ $=1 /\left(L_{\perp}^{2} v_{\perp}^{2}\right) \sum_{j=1}^{N} \delta\left(x-X_{j}\right) \delta\left(v-V_{j}\right)$, where $L_{\perp}$ and $v_{\perp}$ formally represent the (transverse) scales of the system in configura- 
tion and velocity space. The beam density $n_{b}$ is related to $N$ by $N=n_{b} L_{\perp}^{2} L$, where $L$ denotes the assumed periodicity length of the system along $x$. Under the simplifying assumption of a single wave mode, the self-consistent dynamics of the system is described by the following time independent Hamiltonian with $N+1$ degrees of freedom:

$$
\mathcal{H}\left(\theta_{j}, P_{j}, \psi, I\right)=\frac{1}{2} \sum_{j=1}^{N} P_{j}^{2}-2 \sqrt{\frac{I}{N}} \sum_{j=1}^{N} \cos \left(\theta_{j}-\psi\right),
$$

where all variables are dimensionless, the canonical momenta $P_{j}$ are related to the particle velocities $V_{j}$ by $P_{j}$ $=\beta^{-2 / 3}\left(k V_{j}-\omega_{0}\right) / \omega_{p}$, and the phases $\theta_{j}$ to particle positions $X_{j}$ by $\theta_{j}=k X_{j}$, where $k=2 \pi / L, \beta^{2}=n_{b} /\left(n_{0} \omega_{p}\left|\partial_{\omega} \epsilon_{0}\right|\right)$, and $\omega_{0}$ the solution of the relevant dispersion relation $\epsilon(k, \omega)=0$, with $\partial_{\omega} \epsilon_{0}=(\partial \epsilon / \partial \omega)_{\omega=\omega_{0}}$. The action-angle variables $I, \psi$ are related to the dimensional electric field $E$ by $E$ $=2 \beta^{4 / 3} \omega_{p}^{2} m / e k(I / N)^{1 / 2} \sin (k x-\psi)$. The time variable relevant to the Hamiltonian (1) is $\tau=t \omega_{p} \beta^{2 / 3}$. The essential features of the fully nonlinear dynamics of the system are retained in this model, even if a single wavelength is taken into account $[1,7]$. The Hamiltonian (1) has the following constant of motion:

$$
\sum_{j} P_{j}+I=\sum_{j} P_{j 0}+I_{0}=N \Delta,
$$

where $\Delta$ is a constant. Here, we are interested in the time behavior of the system for a specific initial condition, i.e., a small field perturbations $\left(I_{0} / N \approx \epsilon \ll 1\right)$, and an almost monochromatic beam. In this case, the parameter $N \Delta$ represents the initial momentum of the beam in the wave frame, and $\Delta \approx\left[2 n_{0} / n_{b}\left(\omega / \omega_{p}\right)\right]^{1 / 3}\left(V_{b} / V_{\mathrm{ph}}-1\right)$, where $V_{\mathrm{ph}}$ is the phase velocity of the plasma wave for the case under consideration. It is found that the behavior of this system critically depends on $\Delta$ [1]. When $\Delta$ is larger than the critical value $\Delta_{c}$ $=3 / 2^{2 / 3} \approx 1.89$, the field amplitude remains very small during the whole evolution of the system, and the beam electrons move very close to their unperturbed orbits. When $\Delta<\Delta_{c}$ (i.e., at lower beam velocity or/and higher beam density) a considerable fraction of the beam energy can be transferred to the plasma waves. This specific behavior of the system has been observed and analyzed in detail for the Compton regime of a free-electron laser (see, e.g., Ref. [4], and references therein).

Assuming that the electric field is small and behaves as $\exp (i \lambda \tau)$, the following dispersion relation can be obtained from the linearized dynamics of the system (1) around the given initial condition [4]

$$
\lambda^{3}-\Delta \lambda^{2}+1=0 .
$$

For $\Delta>\Delta_{c}$, Eq. (3) has three real solutions corresponding to stable modes, while for $\Delta \leqslant \Delta_{c}$, there are one real and two complex conjugate solutions, and the field evolution is described by the proper superposition of the three linear modes. Quite naturally, the perturbative analysis of the system describes correctly the evolution of the system as long as the field is small. In the unstable case, the results of the analysis are valid for short time, and fail in the asymptotic limit due to the presence of the exponentially growing solution. Three

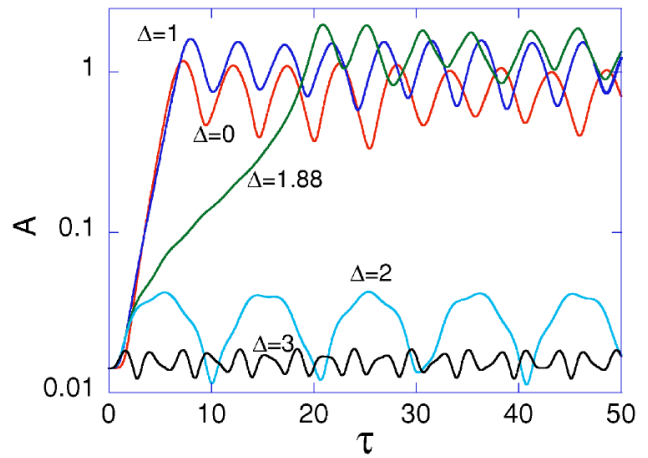

FIG. 1. (Color online) Time behavior of the field amplitude $A$ $\equiv(I / N)^{1 / 2}=\left(\Delta-\Sigma_{j} P_{j} / N\right)^{1 / 2}$ for different values of $\Delta$.

stages can be identified in the time evolution of the field. Initially, there is a lethargy stage (for $\tau \lesssim|\operatorname{Im}(\lambda)|^{-1}$ ), in which the field amplitude is determined by all the three roots of the dispersion, then the field grows exponentially as $\exp [|\operatorname{Im}(\lambda)| \tau]$, and finally it saturates and oscillates around a finite value. The time behavior of the normalized field amplitude $A$, with $A \equiv(I / N)^{1 / 2}=\left(\Delta-\Sigma_{j} P_{j} / N\right)^{1 / 2}$ solution of the Hamilton equations derived by Eq. (1), is shown in Fig. 1 for different values of $\Delta$, corresponding to stable and unstable cases. It can be observed that the linear solution is also valid for almost the whole exponential phase in the unstable regime.

We now consider the nonlinear investigation of the system. From Eq. (1) by means of a canonical transformation, and using the constant of motion, one obtains a $N$ degrees of freedom Hamiltonian

$$
H\left(q_{j}, p_{j}\right)=\frac{1}{2} \sum_{j=1}^{N}\left(p_{j}+\Delta\right)^{2}-2 \sqrt{-\langle p\rangle} \sum_{j=1}^{N} \cos q_{j},
$$

where $p_{j}=P_{j}-\Delta, q_{j}=\theta_{j}-\psi$, the symbol $\langle\cdots\rangle=\Sigma_{i=1}^{N}(\cdots) / N$ denotes average over the $N$ particles, and the constant of motion now reads $I+\Sigma p_{j}=0$. The relevant Hamilton equations read

$$
\begin{gathered}
\dot{q}_{j}=\partial H / \partial p_{j}=p_{j}+\Delta+\langle\cos q\rangle / \sqrt{-\langle p\rangle}, \\
\dot{p}_{j}=-\partial H / \partial q_{j}=-2 \sqrt{-\langle p\rangle} \sin q_{j} .
\end{gathered}
$$

The Hamiltonian (4) can be defined as a "paradigmatic" Hamiltonian for many different physical systems with a selfconsistent dynamics. It describes $N$ pendulums nonlinearly coupled, where the self-consistent field has the amplitude $A$ $=(-\langle p\rangle)^{1 / 2}$.

To get a further understanding of the main features of the system, we present here the results relevant to the phase space topology of the Hamiltonian (4), which allows one to point out the global, long lasting characteristic of the system. It is found [4] that the phase space is characterized by the presence of a large number of stationary points of mixed nature in the $2 N$-dimensional phase space $\left(\geqslant 2^{N-1}\right.$, the exact number depending on the value of $\Delta$ ). Among all these points, there is an elliptic point of coordinates $q_{j}=0, p_{j}=p_{e}$ $(j=1, N)$ for any $\Delta$ value, and a hyperbolic point, character- 

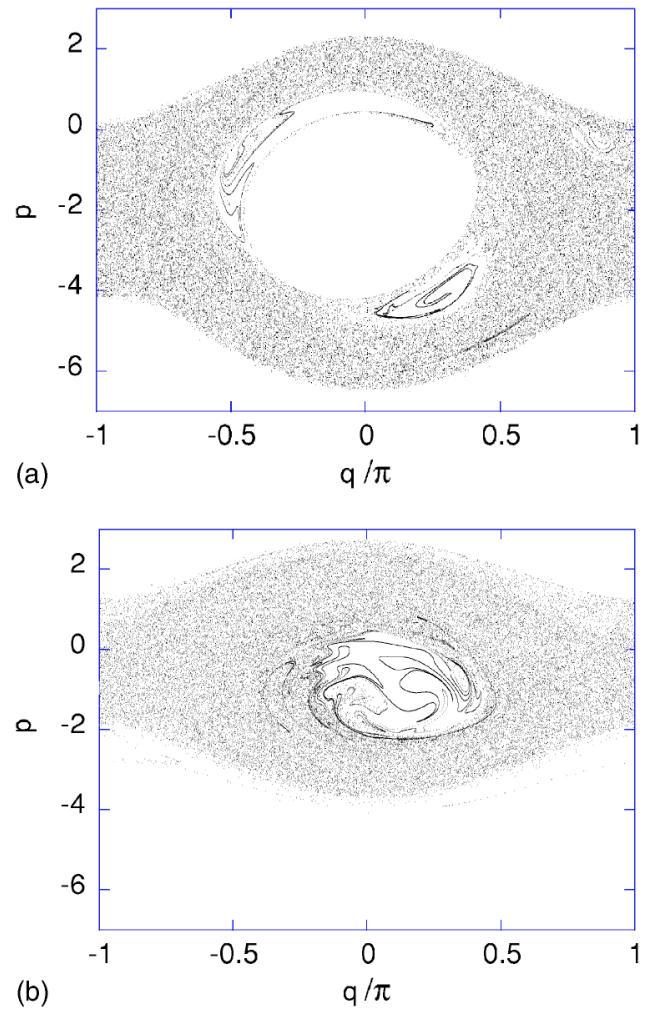

FIG. 2. 2D phase space portrait of late time evolution of the beam particles in an unstable case, for $N=10^{5}, \tau=200$, and two different $\Delta$ values. Case (a) corresponds to $\Delta=1.88$ and case (b) to $\Delta=0$. The coordinates of the elliptic point are $q_{j}=0, p_{j}=p_{e}=-2.51$, and $q_{j}=0, p_{j}=p_{e}=-1$, respectively. The initial distribution corresponds to a monochromatic beam, i.e., uniform in $q$ and $p_{j}=-2$ $\times 10^{-4}$.

ized by $q_{j}=\pi, p_{j}=p_{h},(j=1, N)$ only for $\Delta \geqslant \Delta_{c}$. In the above expressions $p_{e}$ and $p_{h}$ are solutions of the equation

$$
\Lambda^{3}-\Delta \Lambda-\alpha=0
$$

with $\Lambda \equiv(-p)^{1 / 2}$, and $\alpha \equiv\langle\cos q\rangle= \pm 1$, respectively. Note that $\Lambda$ corresponds to the field amplitude of a system in which all the particles are in the fixed point(s). Comparing Eqs. (3) and (6), it is found that the transition to the linearly unstable regime $\left(\Delta \leqslant \Delta_{c}\right)$ corresponds to the occurrence of a topological change in phase space, where the only hyperbolic point disappears.

A representation of the phase space region where the motion of the system occurs is given in Fig. 2, where the particle coordinates $\left(q_{j}, p_{j}\right)$ are plotted in a $2 \mathrm{D}$ space $(q, p)$, after a large number of nonlinear oscillations in the unstable regime, for two different $\Delta$ values. The initial monochromatic distribution evolves and in the nonlinear saturated stage spreads in phase space around the elliptic point determined by Eq. (6). The formation of a hole in the single particle phase space can be observed, which is quite large for $\Delta$ values close to the critical value $\Delta_{c}$. For decreasing $\Delta$ values, the hole in phase space shrinks, and the presence of a well definite bunch of particles becomes more apparent. This bunch rotates in time around the elliptic point, and is mainly

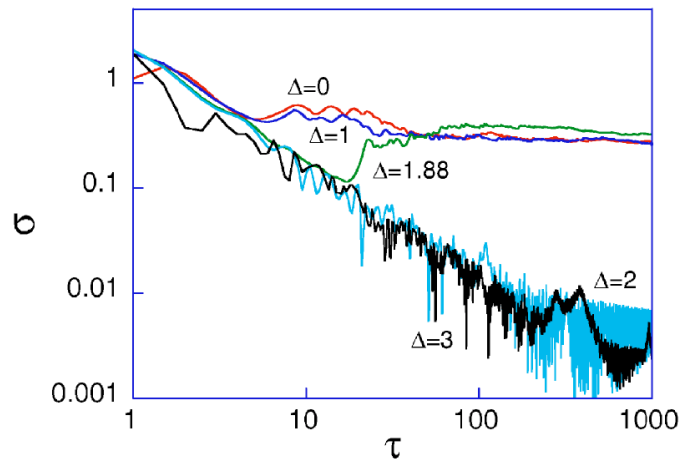

FIG. 3. (Color online) Time behavior of the finite time maximum Lyapunov exponent $\sigma$ for $N=50$, and the same $\Delta$ values of Fig. 1.

responsible for the nonlinear field oscillations, as it has been pointed out in Ref. [3].

Note that this kind of plots are not Poincaré sections, being obtained just as a projection of the full $N$ degrees of freedom dynamics on the $(q, p)$ plane. The investigation of such plots does not, in general, allow one to draw definite statements about the stochasticity of a system. Even a integrable $N$-dimensional system, such as, e.g., that of $N$ uncoupled pendulums, can produce similar plots. Therefore, a more detailed analysis of the stochastic properties of the system described by the Hamiltonian (4) is required.

\section{STOCHASTICITY AND THE ROLE OF DISSIPATION}

To investigate the chaotic dynamics of the system described by the Hamiltonian (4), the maximum Lyapunov exponent $\sigma_{\infty}$ (as defined, e.g., in Ref. [8]) has been numerically computed for the given initial condition, following the method outlined by Wolf et al. in Ref. [9]. The used numerical procedure also determines at any time the finite time Lyapunov exponent $\sigma(\tau)$, which tends asymptotically to $\sigma_{\infty}$. The time evolution of $\sigma(\tau)$ is shown in Fig. 3, for different $\Delta$ values in a range close to $\Delta_{c}$.

For $\Delta$ values corresponding to the stable regime $\left(\Delta>\Delta_{c}\right)$, it is found that $\sigma$ decays in time asymptotically following a power law $\sigma \propto \tau^{-\alpha}$, with $\alpha$ of order unity, indicating the absence of stochasticity in the considered system. In the case of the unstable regime $\left(\Delta<\Delta_{c}\right)$, during the lethargy stage the finite time Lyapunov exponent $\sigma$ decays as in the stable case, then in the exponential stage of the motion it increases sharply, and when the nonlinear oscillating regime (at large amplitude) is established it finally saturates. Asymptotically in time, $\sigma$ tends to a finite value $\sigma_{\infty}$, which is almost independent of $\Delta$. Tests have been made for $N$ values up to 200 . The numerically obtained value of $\sigma_{\infty}$ is about 0.3 , and depends slightly on $N$ and on the final $\tau$. The value of $\sigma_{\infty}$ can be assumed as an index of the degree of mixing of the phase space region where the motion of the system occurs. We have thus shown that the observed sharp variation of the finite-time Lyapunov exponent is related to the phase transition in the $2 N$-dimensional phase space of the system.

We have then investigated the role of weakly dissipative effects, noting that they can allow a stable condition to be- 
come unstable during the evolution of the system, since the Hamiltonian constraints are relaxed. In our investigation, we have first considered radiation damping, as a generic process which alters the Hamiltonian dynamics. It denotes the reaction force that is included in the motion equation of the particle to take into account the fact that energy is being carried out from the system by the waves. It has been shown to play an important role in different fields, such as, e.g., in accelerator physics, in gravitation physics, in the long time evolution of Vlasov-Poisson system [10], in the dynamics of earthquakes and brittle failures of materials where seismic waves or phonons are radiated [11], and in general in systems where Hamiltonians similar to this one can be used. Note that its presence does not necessarily require emission of electromagnetic waves from the system. Here radiation damping is used as a paradigm of dissipative effects.

To include these effects in the Hamiltonian description, we modify the momentum equation, changing $\dot{p}_{j}$ in $\dot{p}_{j}-\eta \ddot{p}_{j}$, where $\eta$ is a constant parameter and $\ddot{p}_{j}$ is computed differentiating Eq. (5b), and using the system (5). This way of computing the radiation damping, which is in general approximate, turns to be exact in the electromagnetic case [12]. Then, instead of system (5), the following equations are obtained:

$$
\begin{gathered}
\dot{q}_{j}=p_{j}+\Delta+\langle\cos q\rangle / A, \\
\dot{p}_{j}=-2 A \sin q_{j}-2 \eta f\left(q_{j}, p_{j}, A\right), \\
\dot{A}=\langle\sin q\rangle,
\end{gathered}
$$

where

$f\left(q_{j}, p_{j}, A\right)=\langle\sin q\rangle \sin q_{j}+\langle\cos q\rangle \cos q_{j}+\left(p_{j}+\Delta\right) A \cos q_{j}$.

Note that "momentum" conservation does not hold anymore.

To point out the main effects of the dissipation term, we use arbitrary $\eta$ values here, which allow one to describe important modifications of the motion in a "short" computer time, while being always small enough to describe a slowly varying perturbation of the Hamiltonian dynamics.

It is found that initially stable conditions evolve towards instability at long times, and amplification of the electric field can occur, as it is shown in Fig. 4, where the time evolution of the amplitude $A$ is plotted for a conservative stable case $(\eta=0)$ and for a slightly dissipative case $(\eta$ $\ll 1)$ for the same initial value of the parameter $\Delta>\Delta_{c}$. Note that the nonlinear field oscillations in the saturated regime are damped by the "dissipation term," and that the system in the $2 N$ phase space collapses in the elliptic point asymptotically in time.

The transition to the high-field regime is again accompanied by a sharp modification of the time evolution of the finite time maximum Lyapunov exponent, which is shown in Fig. 5. In the dissipative case $(\eta>0), \sigma$ initially behaves as in the conservative case $(\eta=0)$, then jumps to a higher value when the field amplitude increases, similarly to what ob-

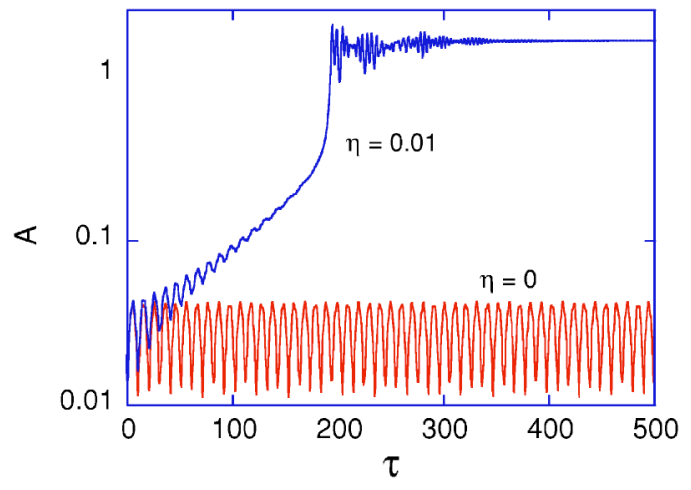

FIG. 4. (Color online) Time behavior of the normalized field amplitude $A=(I / N)^{1 / 2}$ at $\Delta=2$ with $(\eta=0.01)$ and without $(\eta=0)$ dissipation.

served in the unstable conservative case for the corresponding field amplitudes, and finally on the long time scale it slowly decays.

To test the generic validity of our results, we have considered also other different dissipation terms, such as, e.g., a friction force term to mimic the collisional drag on beam electrons. Although the detailed dynamics is different in the two cases (being mainly dependent on the specific choice of the dissipative term), quite similar results have been obtained concerning the transition from the stable to the unstable regime, and the related behavior of the finite time Lyapunov exponent. Again a sudden rise in the value of $\sigma(\tau)$ occurs when the wave amplitude $A$ becomes finite. We then conclude that the transition to the unstable regime can develop from conditions for which the system is stable (i.e., when the initial beam energy is too large to trigger the instability), if it is brought naturally to favorable conditions by dissipative processes.

\section{CONCLUDING REMARKS}

We have presented a generic case of a Hamiltonian system with many degrees of freedom where, starting from a perturbed equilibrium state, the transition to a self-consistent nonlinear equilibrium characterized by large amplitude

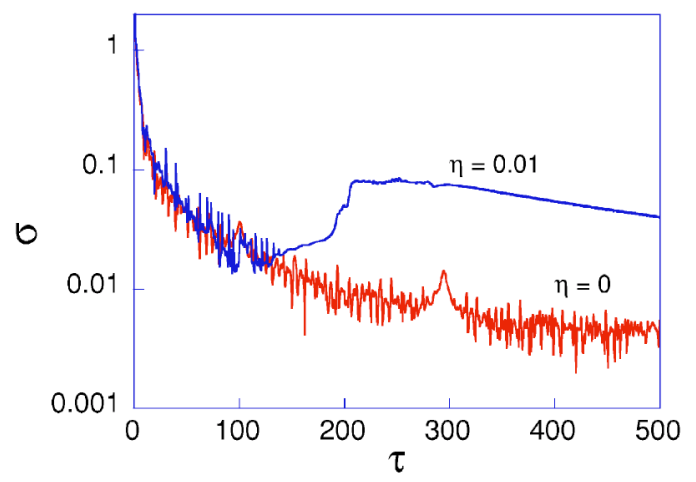

FIG. 5. (Color online) Time behavior of the finite time maximum Lyapunov exponent $\sigma$ for the same parameters of Fig. 4. 
nonlinear oscillations is always connected to an increase of stochasticity $\left(\sigma_{\infty}>0\right)$ and have correlated this process to the evolution of the time-dependent maximum Lyapunov exponent $\sigma(\tau)$. We have shown that such transition can also occur in a otherwise stable system if a weakly dissipative mechanism, as radiation damping or friction, is taken into account, and that it is marked by a sharp variation of $\sigma(\tau)$, as in the Hamiltonian case.
[1] T. M. O’Neil, J. Winfrey, and J. H. Malmberg, Phys. Fluids 14, 1204 (1971).

[2] H. E. Mynick and A. N. Kaufman, Phys. Fluids 21, 653 (1978).

[3] J. L. Tennyson, J. D. Meiss, and P. J. Morrison, Physica D 71, 1 (1994).

[4] D. Farina, F. Casagrande, U. Colombo, and R. Pozzoli, Phys. Rev. E 49, 1603 (1994).

[5] T. Tsuchiya, T. Konishi, and N. Gouda, Phys. Rev. E 53, 2210 (1996).

[6] D. del Castillo-Negrete, Chaos 10, 75 (2000).
[7] D. F. Escande, S. Zekri, and Y. Elskens, Phys. Plasmas 3, 2534 (1996).

[8] A. J. Lichtenberg and M. A. Lieberman, Regular and Stochastic Motion (Springer-Verlag, Berlin, 1983).

[9] A. Wolf, J. B. Swift, H. L. Swinney, and J. A. Vastano, Physica D 16, 285 (1985).

[10] M. Kunze and A. D. Rendall, Ann. Henri Poincare 2, 857 (2001).

[11] A. Johansen and D. Sornette, Phys. Rev. Lett. 82, 5152 (1999).

[12] G. W. Ford and R. F. O'Connell, Phys. Lett. A 174, 182 (1993). 